International Journal of Pure and Applied Mathematics

Volume 106 No. 1 2016, 307-316

ISSN: 1311-8080 (printed version); ISSN: 1314-3395 (on-line version)

url: http://www.ijpam.eu

doi: 10.12732/ijpam.v106i1.25

\title{
THE FIBONACCI NUMBERS FOR THE MOLECULAR GRAPHS OF LINEAR PHENYLENES
}

\author{
Jaroslav Seibert ${ }^{1 \S}$, Libor Koudela ${ }^{2}$ \\ ${ }^{1,2}$ Institute of Mathematics and Quantitative Methods \\ Faculty of Economics and Administration \\ University of Pardubice \\ Studentská 84, 53210 Pardubice, CZECH REPUBLIC
}

\begin{abstract}
The concept of the Fibonacci number of an undirected graph $G=(V, E)$ refers to the number of independent vertex subsets $U$ of $V$ such that no two vertices from $U$ are adjacent in $G$. In this paper the Fibonacci numbers of molecular graphs corresponding to one type of phenylenes are calculated using the decomposition formula. Investigation of the Fibonacci numbers of certain classes of graphs leads to a difference equation or systems of difference equations. The explicit formula for the Fibonacci numbers of linear phenylenes is found as a function of the number $n$ of hexagons in the phenylene.
\end{abstract}

AMS Subject Classification: 92E10, 11B39, 05C90

Key Words: molecular graph, Fibonacci number, linear phenylene, decomposition formula, difference equation

\section{Introduction}

The Fibonacci numbers $F_{n}$ are defined by the second order recurrence $F_{n+2}=$ $F_{n+1}+F_{n}$ with $F_{0}=0, F_{1}=1$. The Lucas numbers $L_{n}$ satisfy the same recurrence with the initial terms $L_{0}=2, L_{1}=1$. All graphs discussed in this paper are undirected simple graphs, which means graphs without loops

Received: December 4, 2015

Published: February 5, 2016

$\S_{\text {Correspondence author }}$ (c) 2016 Academic Publications, Ltd.

url: www.acadpubl.eu 
and multiple edges. For the general graph-theoretic terminology we refer the reader to any of standard monographs, e.g. [11]. The total number of subsets of $\{1,2, \ldots, n\}$ such that no two elements are adjacent is the Fibonacci number $F_{n+2}$. In view of this fact Prodinger and Tichy introduced in 1982 the notion of the Fibonacci number of a graph [8, p. 16].

Definition 1. Let $G-(V, E)$ be a simple graph. The Fibonacci number $f(G)$ of $G$ is defined as the number of all subsets $U$ of $V$ such that no two vertices in $U$ are adjacent.

The subset $U$ of $k$ mutually independent vertices is called the $k$-independent set of $G$. We denote $i(G, k)$ the number of the $k$-independent sets of $G$ and $i(G, 0)=1$ by definition for any graph $G$. Then, the Fibonacci number of $G$ is given by the relation $f(G)=\sum_{k} i(G, k)$, where the summation is taken over all nonnegative integers $k$.

Further, Prodinger and Tichy derived some basic and effective results.

Theorem 1. Let $G_{1}-\left(V, E_{1}\right), G_{2}-\left(V, E_{2}\right)$ be graphs such that $E_{1} \subseteq E_{2}$. Then $f\left(G_{1}\right) \geq f\left(G_{2}\right)$.

Theorem 2. If $G_{1}, G_{2}$ are disjoint graphs, then $f\left(G_{1} \sqcup G_{2}\right)=f\left(G_{1}\right) f\left(G_{2}\right)$.

Theorem 3. Let $P_{n}$ be a path with $n$ vertices and $C_{n}$ a circuit with $n$ vertices. Then $f\left(P_{n}\right)=F_{n+2}$ and $f\left(C_{n}\right)=L_{n}$.

The following decomposition theorems (see e.g. [7]) can be used to calculate the Fibonacci numbers for special classes of graphs.

Theorem 4. Let $G$ be a graph with at least two vertices and $v$ its arbitrary vertex. Then $f(G)=f(G-v)+f(G-(v))$, where $G-v$ is the subgraph of $G$ obtained from $G$ by deletion of the vertex $v$ and $G-(v)$ is the subgraph of $G$ obtained by deletion of the vertex $v$ and all the vertices adjacent to $v$.

Theorem 5. If vertices $u, v$ are adjacent in a graph $G$, then

1. $f(G)=f(G-\{u, v\})+f(G-(u))+f(G-(v))$, where $G-\{u, v\}$ is the subgraph of $G$ obtained by deletion of the vertices $u$ and $v$ of $G$, 
2. $f(G)=f(G-u v)-f(G-(u, v))$, where $G-u v$ is the subgraph of $G$ obtained by deletion of the edge $u v$ of $G$ and $G-(u, v)$ is the subgraph of $G$ obtained by deletion of the vertices $u, v$ and all the vertices adjacent to them.

The Fibonacci numbers for various classes of graph have been found. For example, Yeh [12] computed algorithmically the Fibonacci numbers of product graphs and Alameddine [1] found upper and lower bounds for the Fibonacci numbers of maximal outerplanar graphs on $n$ vertices. We presented in [9] a computer program for calculating of the Fibonacci number for a given graph. This program was created by using the adjacency matrix of a graph and an analogy of Theorem 4. A special attention is paid to the Fibonacci numbers of graphs in chemistry.

A molecular graph in chemical graph theory is a representation of the structural formula of a chemical compound in terms of graph theory. A topological index is a map from the set of chemical compounds represented by molecular graphs to the set of real numbers. It must be a structural invariant independent on the pictorial representation of a graph. Many topological indices are closely related to some physico-chemical characteristics of the respective compounds. One of the most famous and interested topological indices is the Fibonacci number of a molecular graph. The chemists Merrifield and Simmons elaborated a theory aimed at describing molecular structure by means of finite set topology. Even though their theory was not particularly succesful their graph-topological formalism containing independent sets of vertices attracted the attention of colleagues. Therefore in chemistry the Fibonacci number of a graph is called the Merrifield-Simmons index. In this paper we prefer to use the term 'Fibonacci number of a graph'.

Hexagonal systems are of the great importance for theoretical chemistry because they are the natural graph representations of the benzenoid hydrocarbons [5]. Hexagonal chains are the graph representations of an important subclass of benzenoid molecules, namely of the so-called unbranched cata-condensed benzenoids. The structure of these graphs is apparently the simplest among all hexagonal systems [6]. Therefore the first results on topological indices were achieved for hexagonal chains. Došlić and Litz [4] investigated enumerative properties of unbranched polyphenylene chains. They found exact formulas for the numbers of independent sets of given cardinalities in three types of uniform chains and also presented some results on polyphenylene dendrimers. Bai and Zhao [3] discussed the Merrifield-Simmons index of polyphenyl chains and obtained some extremal results. In recent years, a lot of works have been pub- 
lished on the extremal problem for the Fibonacci number of graphs. Wagner and Gutman in [10] gave a survey which collects and classifies these results, and also provides some useful auxiliary tools and techniques that are used in the study of this type of problems.

Phenylenes are a class of polycyclic non-benzenoid alternate conjugated hydrocarbons in that the carbon atoms form 6- and 4- membered circuits (cycles). Each 4- membered circuit (square) is adjacent to two disjoined 6- membered circuits (hexagons), and no two hexagons are adjacent. Their respective molecular graphs are also referred to as phenylenes.

By eliminating the 4- membered circuits from a phenylene, a cata-condensed hexagonal system is obtained, sometimes called as the hexagonal squeeze of the respective phenylene. There is a one-to-one correspondence between a phenylene and its hexagonal squeeze [2]. Gutman [5] pointed out that the linear hexagonal chain is the unique hexagonal chain with the maximum MerrifieldSimmons index among all the hexagonal chains with $n$ hexagons. The linear phenylene $L_{n}$ (see Fig. 1) has the linear hexagonal chain as its squeeze. Therefore it is likely that the Fibonacci number of the linear phenylene is the maximum Fibonacci number among all the phenylenes with $n$ hexagons. We will express the Fibonacci number of the linear phenylene as a function of the number of its hexagons in the next section.

\section{The Main Results}

First, we derive the system of equalities for the Fibonacci numbers of the graphs obtained from the linear phenylene using the suitable decomposition theorem.

Lemma 1. Let $L_{n}$ be the linear phenylene with $n$ hexagons and let $A_{n}$, $B_{n}, D_{n}, E_{n}$ be graphs as in Fig. 1. Then the following relations hold for any positive integer $n>1$.

$$
\begin{gathered}
f\left(L_{n}\right)=f\left(A_{n}\right)+f\left(D_{n}\right) \\
f\left(A_{n}\right)=f\left(B_{n}\right)+f\left(D_{n}\right) \\
f\left(B_{n}\right)=4 f\left(L_{n-1}\right)+4 f\left(A_{n-1}\right) \\
f\left(D_{n}\right)=f\left(L_{n-1}\right)+f\left(A_{n-1}\right)+f\left(E_{n}\right) \\
f\left(E_{n}\right)=f\left(L_{n-1}\right)+2 f\left(A_{n-1}\right)
\end{gathered}
$$



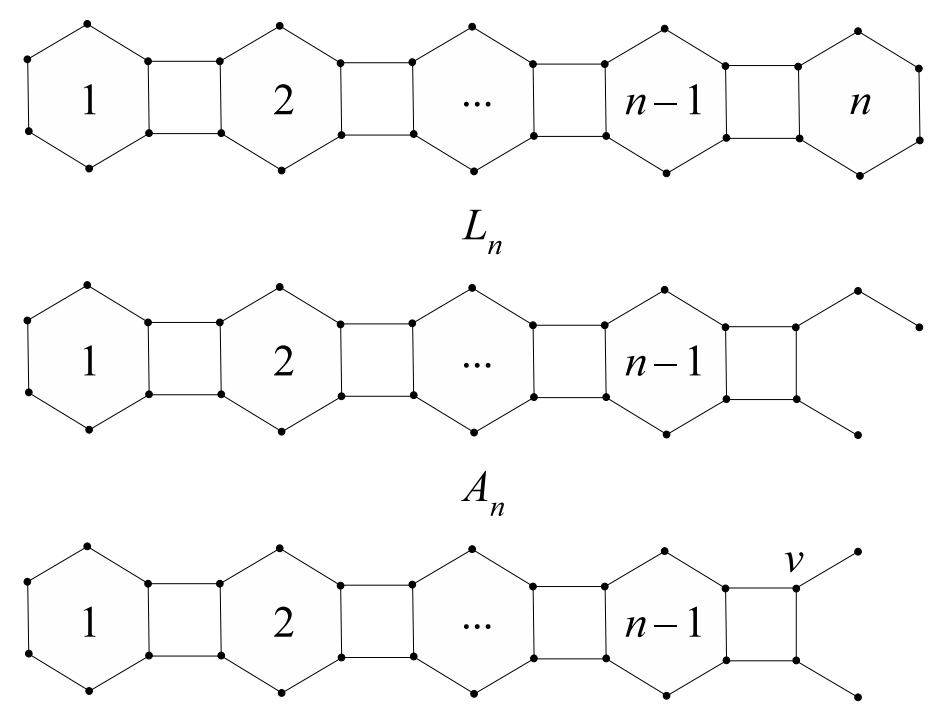

$B_{n}$

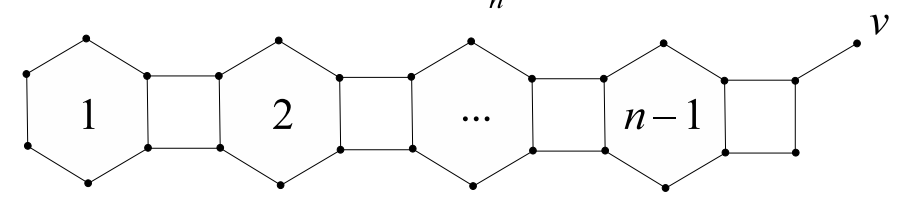

$D_{n}$

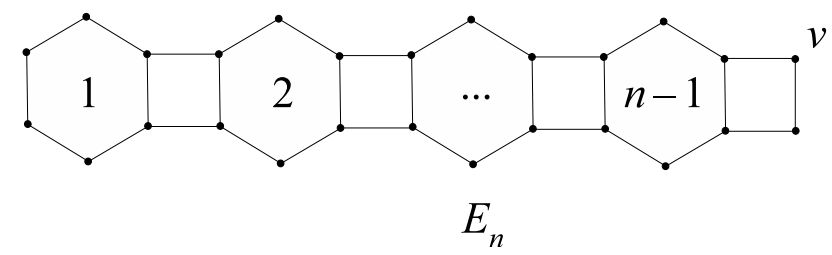

Figure 1: The molecular graph of the linear phenylene

Proof. Each relation can be derived by using Theorem 4 if we choose the removed vertex $v$ in a suitable way (see Fig. 1). Specifically we put

(1) vertex $v$ to one of vertices of degree 2 in the $n$-th hexagon which is adjacent to two vertices of degree 2 ,

(2) vertex $v$ to the vertex of degree 1 which is adjacent to the vertex of degree 2 ,

(3) vertex $v$ to one of vertices of degree 3 in the last square which is adjacent 
to the vertex of degree 1 , and then we use again Theorem 4 on the graph $B_{n}-(v)$ and Theorem 2, with the fact that $f\left(P_{1}\right)=F_{3}=2$, which gives desired expression,

(4) vertex $v$ to the vertex of degree 1 and after that we apply Theorem 4 on the graph $D_{n}-(v)$ where the vertex of degree 1 is chosen,

(5) vertex $v$ to one of vertices of degree 2 in the last square.

Relations (1)-(5) are also valid for $n=1$, as the Fibonacci number of the empty graph is equal to one. Now, we denote $f\left(L_{n}\right)=l_{n}, f\left(A_{n}\right)=a_{n}$, $f\left(B_{n}\right)=b_{n}, f\left(D_{n}\right)=d_{n}$ and $f\left(E_{n}\right)=e_{n}$ for simplification of the following text.

Theorem 6. The Fibonacci numbers of the graphs $A_{n}$ are given in the form

$$
f\left(A_{n}\right)=a_{n}=\frac{1}{\gamma-\delta}\left[(199-13 \delta) \gamma^{n-1}-(199-13 \gamma) \delta^{n-1}\right]
$$

for any positive integer $n$, where $\gamma=\frac{15+^{\vee} \overline{241}}{2}, \delta=\frac{15-^{\vee} \overline{241}}{2}$.

Proof. First, we will find a linear difference equation arising from Lemma 1 with $a_{n}$ as the unknown variable. We can rewrite the system of relations (1)-(5) to the form

$$
\begin{gathered}
l_{n}=a_{n}+d_{n}, \\
a_{n}-d_{n}=4 l_{n-1}+4 a_{n-1}, \\
d_{n}=2 l_{n-1}+3 a_{n-1},
\end{gathered}
$$

and after eliminination of $d_{n}$

$$
\begin{gathered}
2 a_{n}=l_{n}+4 l_{n-1}+4 a_{n-1}, \\
a_{n}=6 l_{n-1}+7 a_{n-1} .
\end{gathered}
$$

We obtain $l_{n-1}=\frac{1}{6} a_{n}-\frac{7}{6} a_{n-1}$ from equality (7) and replacing $n-1$ with $n$ we have $l_{n}=\frac{1}{6} a_{n+1}-\frac{7}{6} a_{n}$.

Substituting these identities for $l_{n-1}$ and $l_{n}$ into (6) and after simplification we obtain $a_{n+1}-15 a_{n}-4 a_{n-1}=0$ which can be also written as

$$
a_{n+2}-15 a_{n+1}-4 a_{n}=0
$$

for any positive integer $n$. 
The last equation is a homogeneous linear difference equation of the second order with constant coefficients. The corresponding characteristic equation is $x^{2}-15 x-4=0$ with two real roots $\gamma=\frac{15+\overline{241}}{2}, \delta=\frac{15-\overline{241}}{2}$.

The general solution of equation (8) is $a_{n}=K_{1} \gamma^{n}+\stackrel{2}{K_{2}} \delta^{n}$, where $K_{1}, K_{2}$ are arbitrary real numbers. Using Theorems 2,3 and 4 we can easily calculate

$$
a_{1}=f\left(P_{5}\right)=13, \quad a_{2}=2\left(f\left(P_{8}\right)+f^{2}\left(P_{3}\right)\right)+3 f\left(P_{5}\right)=199 .
$$

Then, we obtain the following system of equations for $K_{1}, K_{2}$

$$
\begin{gathered}
K_{1} \gamma+K_{2} \delta=13, \\
K_{1} \gamma^{2}+K_{2} \delta^{2}=199 .
\end{gathered}
$$

The numbers $K_{1}=\frac{199-13 \delta}{\gamma(\gamma-\delta)}, K_{2}=\frac{199-13 \gamma}{\delta(\gamma-\delta)}$ are the solution of this system and therefore $a_{n}=\frac{199-13 \delta}{\gamma(\gamma-\delta)} \gamma^{n}+\frac{199-13 \gamma}{\delta(\gamma-\delta)} \delta^{n}$, which completes the proof.

The following statement gives the main result of this paper.

Theorem 7. The Fibonacci number of the linear phenylene with $n$ hexagons can be expressed in the form

$$
f\left(L_{n}\right)=l_{n}=\frac{1}{\gamma-\delta}\left[(18 \gamma+4) \gamma^{n-1}-(18 \delta+4) \delta^{n-1}\right]
$$

for any positive integer $n$.

Proof. We know that $l_{n}=\frac{1}{6} a_{n+1}-\frac{7}{6} a_{n}$. Then successively

$$
\begin{aligned}
l_{n}= & \frac{1}{6(\gamma-\delta)}\left[(199-13 \delta) \gamma^{n}-(199-13 \gamma) \delta^{n}\right]- \\
& -\frac{7}{6(\gamma-\delta)}\left[(199-13 \delta) \gamma^{n-1}-(199-13 \gamma) \delta^{n-1}\right]= \\
= & \frac{1}{6(\gamma-\delta)}\left[(\gamma-7)(199-13 \delta) \gamma^{n-1}-(\delta-7)(199-13 \gamma) \delta^{n-1}\right]= \\
= & \frac{1}{6(\gamma-\delta)}\left[(108 \gamma+24) \gamma^{n-1}-(108 \delta+24) \delta^{n-1}\right]
\end{aligned}
$$

as $\gamma \delta=-4$ and $\gamma+\delta=15$, which gives the desired expression.

The leading term of the above expression behaves asymptotically as $15,262^{n}$. Therefore, we can conclude that $f\left(L_{n}\right) \sim 15,262^{n}$ for large values of $n$. 


\begin{tabular}{|c|c|c|c|c|c|c|c|}
\hline$n$ & 1 & 2 & 3 & 4 & 5 & 6 & 7 \\
\hline$f\left(L_{\mathrm{n}}\right)$ & 18 & 274 & 4182 & 63826 & 974118 & 14867074 & 226902582 \\
\hline
\end{tabular}

Table 1: The Fibonacci numbers of $L_{n}$ for $1 \leq n \leq 7$

We have computed the Fibonacci numbers of the linear phenylenes $L_{n}$ for the smallest numbers of hexagons in the linear phenylenes (Tab. 1).

Remark. Obviously the linear recurrences from Lemma 1 lead to the same linear difference equation for sequences $\left\{b_{n}\right\},\left\{d_{n}\right\}$ and $\left\{e_{n}\right\}$ as for $\left\{a_{n}\right\}$. The explicit formulas for them are different only in coefficients $K_{1}, K_{2}$, which can be established by using the initial terms of the sequences, specifically $b_{1}=8$, $b_{2}=124, d_{1}=5, d_{2}=75, e_{1}=3, e_{2}=44$. However, it is also possible to use directly recurrences (3), (4), (5) and the explicit formulas for $a_{n}$ and $l_{n}$. Then we have

$$
\begin{aligned}
& b_{n}=\frac{2}{3(\gamma-\delta)}\left[(\gamma-1)(199-13 \delta) \gamma^{n-2}-(\delta-1)(199-13 \gamma) \delta^{n-2}\right] \\
& d_{n}=\frac{1}{6(\gamma-\delta)}\left[(\gamma-13)(199-13 \delta) \gamma^{n-1}-(\delta-13)(199-13 \gamma) \delta^{n-1}\right] \\
& e_{n}=\frac{1}{6(\gamma-\delta)}\left[(\gamma+5)(199-13 \delta) \gamma^{n-2}-(\delta+5)(199-13 \gamma) \delta^{n-2}\right]
\end{aligned}
$$

These explicit formulas are valid for any positive integer $n$, only if we want to use them to find $b_{1}$ and $e_{1}$ the identities $\gamma^{-1}=-\frac{\delta}{4}, \delta^{-1}=-\frac{\gamma}{4}$ have to be applied.

The above formulas can be simplified by using the relations $\gamma \delta=-4$ and $\gamma+\delta=15$. Then

$$
\begin{aligned}
& b_{n}=\frac{1}{\gamma-\delta}\left[(124 \gamma+32) \gamma^{n-2}-(124 \delta+32) \delta^{n-2}\right] \\
& d_{n}=\frac{5}{\gamma-\delta}\left(\gamma^{n}-\delta^{n}\right) \\
& e_{n}=\frac{1}{\gamma-\delta}\left[(44 \gamma+12) \gamma^{n-2}-(44 \delta+12) \delta^{n-2}\right] .
\end{aligned}
$$

It is also easy to find by a classical way the ordinary generating functions for the sequences of the Fibonacci numbers of the above mentioned graphs. The generating functions for the sequnces $\left\{l_{n}\right\},\left\{a_{n}\right\},\left\{b_{n}\right\},\left\{d_{n}\right\},\left\{e_{n}\right\}$ are successively 


$$
\begin{aligned}
L(x) & =\sum_{n=1}^{\infty} l_{n} x^{n}=\frac{18 x+4 x^{2}}{1-15 x-4 x^{2}} \\
A(x) & =\frac{13 x+4 x^{2}}{1-15 x-4 x^{2}} \\
B(x) & =\frac{8 x+4 x^{2}}{1-15 x-4 x^{2}} \\
D(x) & =\frac{5 x}{1-15 x-4 x^{2}} \\
E(x) & =\frac{3 x-x^{2}}{1-15 x-4 x^{2}} .
\end{aligned}
$$

\section{Conclusion}

The application of Theorem 4 to find explicit formulas (depending on one or two parameters) of the Fibonacci numbers for various classes of graphs leads to a difference equation of a higher order or a system of difference equations. However, the system of difference equations often consists of a great number of equations and then the solution is rather complicated. For example, this situation appears if we want to find an expression for the Fibonacci numbers of some nonlinear types of phenylenes. In such cases it is possible to find at least some bounds for the Fibonacci numbers of given graphs or to express an explicit formula for them in a numerical form. The decomposition formulas from Theorem 5 can be more effective than Theorem 4 for the branched phenylenes.

\section{References}

[1] A.F. Alameddine, Bounds on the Fibonacci Number of a Maximal Outerplanar Graph, Fibonacci Quarterly, 36, No. 2 (1998), 206-210.

[2] S. Aziz, A. Manikpuri, P.E. John, P.V. Khadikar, Computation of the Sadhana index of linear phenylenes and corresponding hexagonal sequences, Iranian Journal of Mathematical Chemistry, 1 (2010), 79-90.

[3] Y. Bai, B. Zhao, P. Zhao, Extremal Merrifield-Simmons index and Hosoya index of polyphenyl chains, MATCH Commun. Math. Comput. Chem., 62 (2009), 649-656.

[4] T. Došlić, M.S. Litz, Matching and independent sets in polyphenylene chains, MATCH Commun. Math. Comput. Chem., 67 (2012), 313-330.

[5] I. Gutman, Extremal hexagonal chains, Journal of Mathematical Chemistry, 12 (1993), 197-210, doi: 10.1007/BF01164635. 
[6] I. Gutman, S.J. Cyvin (eds.), Advances in the Theory of Benzenoid Hydrocarbons, Springer, Berlin (1989).

[7] A.S. Pedersen, P.D. Vestergaard, The number of independent sets in unicyclic graphs, Discrete Applied Mathematics, 152 (2005), 246-256, doi:10.1016/j.dam.2005.04.002.

[8] H. Prodinger, R.F. Tichy, Fibonacci Numbers of Graphs, Fibonacci Quarterly, 20, No. 1 (1982), 16-21.

[9] J. Seibert, P. Trojovský, Recurrences and Generating Functions for Fibonacci Numbers of Graphs with Mathematica, In: Proceedings of the Conference Informatika a algoritmy, Prešov (2002), 36-41.

[10] S. Wagner, I. Gutman, Maxima and minima of the Hosoya index and the MerrifieldSimmons index: A survey of results and techniques, Acta Appl. Math. 112 (2010), 23-346, doi: 10.1007/s10440-010-9575-5.

[11] D.B. West, Introduction to Graph Theory, Prentice Hall (2001).

[12] L. Yeh, Fibonacci Numbers of Product Graphs, JCMCC: The Journal of Combinatorial Mathematics and Combinatorial Computing, 32 (2000), 223-229. 